\title{
The Neurobiology of Behavioral Impulsivity as Expressed in the Hyperactive-Impulsive and Combined Presentations of Attention-Deficit/ Hyperactivity Disorder
}

\author{
Robert Eme* \\ Professor of Clinical Psychology, Illinois School of Professional Psychology at Argosy University at Schaumburg \\ Campus, Schaumburg, Illinois, U.S.A.
}

Received: June 09, 2017; Accepted: June 20, 2017; Published: June 29, 2017

*Corresponding author: Robert Eme, Professor of Clinical Psychology, Illinois School of Professional Psychology at Argosy University at Schaumburg Campus, Schaumburg, Illinois, U.S.A, Tel: 847.969.4943; fax: 847.969.4998; E-mail: reme@argosy.edu

\begin{abstract}
The article provided a mini review of the neuropathology of behavioral impulsivity as expressed in the hyperactive-impulsive and combined presentations of ADHD. The two most important neural circuits subserving behavior impulsivity are the mesolimbic and mesocortical dopamine circuits. A dysfunctional mesolimbic circuit is implicated in the generation of behavioral impulsivity and the mesocortical circuit is implicated in the failure to control behavioral impulsivity. Through complex interaction with various environmental risk factors, the impulsivity subserved by these circuits increases the risk for various antisocial disorders across development.
\end{abstract}

\section{Introduction}

The neuropsychiatric disorder of Attention-deficit/ hyperactivity Disorder (ADHD) is the diagnosis given to juveniles and adults who present with impairing symptoms of attention and/or impulsivity and hyperactivity [1]. Its importance is underscored by the fact that it is one of the most prevalent juvenile disorders as well as one of the most common reasons children with behavioral problems are referred to medical and mental health practitioners in the United States. Its causes are primarily genetic with other variables such as low birth weight, exposure to lead, etc. also contributing to its origin [2]. In addition, it substantially increases the risk for a kaleidoscope of long-term adverse outcomes [4]. Of all the adverse outcomes, the antisocial disorders of Oppositional Defiant Disorder, Conduct Disorder, Substance Use Disorder, and Antisocial Personality Disorder which disproportionately affect males are arguably the most serious because of the consequences they pose for the individual as well as society $[5,6]$.This increased risk of antisocial disorders is primarily driven by the behavioral impulsivity expressed early in life in the hyperactive-impulsive and combined presentations of ADHD [5].Hence a basic grasp of the neurobiology of this manifestation of ADHD is important for understanding how it increases the risk for the development of antisocial behavior.

\section{Neurobiology of Behavioral Impulsivity}

Impulsivity is a multifaceted trait mediated by distinct psychological and neurobiological mechanisms [7].Behavioral impulsivity is characterized by: a) decisions based on a preference for smaller, immediate rewards over larger, delayed rewards, and b) a tendency to make rapid decisions without forethought, adequate consideration of the evidence [5,7].Dopamine is a monoamine neurotransmitter that is implicated in the neural processing of reward-related stimuli such as food, sex, including those associated with drugs of abuse $[8,9]$. Rewards defined scientifically act as positive reinforcers to induce learning, elicit movements towards a desired object, and elicit emotions such as pleasure and desire [10]. Dopamine (DA) projections are divided into four primary neural systems, two of which are relevant to behavioral impulsivity: the mesolimbic and mesocortical $[5,8]$. Several current comprehensive reviews concur in implicating impairments in both neural circuits and their interconnections to one another as well as other brain regions as the central neurobiological mechanisms in generating behavioral impulsivity $[5,7,8,11,12,13,14,15]$. These two circuits are linked to two different aspects of impulsivity: impulse generation and impulse control [12].

\section{Mesolimbic Dopamine Circuit and Impulse Generation}

The subcortical mesolimbic DA projections ascend from the ventral tegmental area to the ventral striatum, including the nucleus accumbens and the ventral regions of the caudate nucleus and putamen [8]. Several lines of research indicate that this circuit is the brain's most important reward system $[5,8]$. For example: a) stimulation of the mesolimbic structures in various ways is so highly reinforcing that animals trained 
through operant condition keep responding to the rewarded stimulation to the neglect of primary reinforces such as food and water, b) mesolimbic neural activity increases during rewardseeking and reward-anticipation behaviors, c) blockage of mesolimbic neural activity eliminates the motivation provided by primary reinforces and stimulant drugs of abuse. These findings prompted the development of several theories that linked impulsivity to individual differences in the activity and reactivity of the mesolimbic structures [5]. Subsequently, extensive neuroimaging research has found reduced mesolimbic activity during the anticipation of reward in individuals with ADHD $[5,8,13]$. This hypofunctioning of the mesolimbic circuit results in an undersensitivity to reinforcing stimuli which is hypothesized to trigger impulsivity in two major ways $[5,8,13]$. First, undersensitivity leads to temporal discounting of reward in that individuals with ADHD find the experience in delay of reinforcement so aversive that they tend to impulsively choose immediate rewards even when an alternative option would result in larger reward after a longer delay. Second, undersensitivity leads to excessive, impulsive reward seeking as individuals with ADHD "experience relatively low hedonic value from pleasurable stimuli and therefore seek more frequent extreme incentives to derive hedonic payoff"[8]. In short, undersensitivity to stimuli that most individuals would find reinforcing results in individuals with ADHD having chronic feelings of anhedonia which they find aversive. To alleviate such feelings, they tend to impulsively seek immediate and frequent reinforcement/gratification.

\section{Mesocortical Dopamine Circuit and Impulse Control}

The mesocortical DA circuit involves neural projections from the ventral tegmental area to cerebral cortex, and the prefrontal cortex (PFC) and the anterior cingulate cortex $[5,8,12,14]$.This circuit is involved with the second aspect of impulsivity, i.e., how impulses are contained, stopped or interrupted [12]. The main substructures of the PFC are the dorsolateral PFC; ventromedial $\mathrm{PFC}$; ventrolateral PFC; the orbitofrontal PFC; and anterior cingulate cortex. Each of these substructures are hypothesized to be somewhat differentially involved in top down impulse control $[14,15]$.

Several lines of research document that a breakdown in optimal function and communication between the PFC and the midbrain region can result in failures to contain, inhibit, interrupt impulsive behavior $[5,12,14,15]$. Structural imaging studies using structural magnetic imaging techniques have found that children with ADHD have significantly smaller overall brain volumes, including the PFC and ACC, than comparison children $[15,16,17]$ Studies of connectivity in the mesocortical circuit using diffusion tensor imaging, resting-state functional connectivity, and taskbased functional connectivity techniques have collectively found weaker connectivity between the $\mathrm{PFC}$ and midbrain region $[15,16,17]$. Lastly, task-based functional MRI studies of ADHD have found reduced responding (e.g., hypoactivation as indicated by reduced blood flow) in this circuit thus implicating hypo connectivity and therefore deficient functioning $[12,14,15,17]$.

\section{Conclusions}

This brief review of the neuropathology of behavioral impulsivity as expressed in the hyperactive-impulsive and combined presentations of ADHD has found that the two most important neural circuits are the mesolimbic and mesocortical DA circuits. A dysfunctional mesolimbic circuit is implicated in the generation of behavioral impulsivity and the mesocortical circuit is implicated in the failure to control behavioral impulsivity. The relationship between this circuits is best understood as the circuits influencing one another rather than constituting a single DA circuit [12]. Through complex interaction with various environmental risk factors, the impulsivity mediated by these circuits increases the risk for various antisocial disorders across development.

\section{References}

1. American Psychiatric Association. Diagnostic and statistical manual of mental disorders 5th ed. Washington, DC. 2013.

2. Barkley RA. Attention-deficit hyperactivity disorder: A handbook for diagnosis \& treatment.3rd ed. New York: Guilford Press. 2015.

3. Owens E, Cardoos S, Hinshaw S. Developmental progression and gender differences among individuals with ADHD. In: Barkley R, editor. Attention-deficit hyperactivity disorder. 4th ed. New York: Guilford Press; 2015. P.223-255.

4. Hechtman L. Attention Deficit Hyperactivity Disorder. New York: Guilford Press; 2017.

5. Beauchaine T, Zisner Ac, Sauder C. Trait impulsivity and the externalizing spectrum. Annual review of Clinical psychology. 2017;13:343-368

6. Eme R. Sex differences in the prevalence and expression of externalizing spectrum disorders. In: Beauchaine TP, Hinshaw SP, editors. Oxford handbook of externalizing spectrum disorders. New York: Oxford University Press; 2016. P. 239-266.

7. Dalley JW, Robbins TW. Fractionating impulsivity: neuropsychiatric implications. Nat Rev Neurosci. 2017;18(3):158-171.

8. Zisner A, Beauchaine T. Neural substrates of trait impulsivity, anhedonia, and irritability: Mechanisms of heterotypic comorbidity between externalizing disorders and unipolar depression. Development and Psychopathology. 2016;28(4pt1):1177-1208.

9. Volkow ND, Morales M. The brain on drugs: from reward to addiction. Cell. 2015;162(4):712-725.

10.Schultz W. Dopamine reward predication-error signaling: a twocomponent response. Nat Rev Neurosci. 2016;17(3):183-195.

11. Petrovic P. Castellanos FX. Top-down Dysregulation-from ADHD to emotional instability. Front Behav Neurosci. 2016;10:70.

12. Nigg JT. Attention and impulsivity. In: Cicchetti D, editor. Developmental psychopathology. 3rd ed. New York: Wiley; 2016. P. 591-646.

13.Zisner A, Beauchaine T. Midbrain mechanisms of trait impulsivity. In: 
Beauchaine T, Hinshaw S, editors. Oxford handbook of externalizing spectrum disorders. New York: Oxford University Press; 2016. P.184200.

14. Ryan NC, Seguin JR. Prefrontal and anterior cingulate cortex mechanisms of impulsivity. In: Beauchaine $\mathrm{T}$, Hinshaw S, editors Oxford handbook of externalizing spectrum disorders. New York: Oxford University Press; 2016. P.184-200.

15. Gallo EF, Posner J. Moving towards causality in attention-deficit hyperactivity disorder. Overview of neural and genetic mechanisms. The Lancet Psychiatry. 2016;3(6):555-567.
16. Rubia K, Alegria AA, Brinson H. Brain abnormalities in attentiondeficit hyperactivity disorder: a review. Rev Neurol. 2014;58: Suppl 1:S3-16

17.Ahmad S, Hinshaw S. Attention-Deficit/Hyperactivity Disorder: Similarities to and differences from other externalizing disorders. In: Beauchaine $\mathrm{T}$, Hinshaw $\mathrm{S}$, editors. The Oxford handbook of externalizing disorders. New York: Oxford Handbooks; 2016.p.19-37. 